\title{
TRES LUSTROS DE LA FACULTAD DE MEDICINA
}

\author{
Dario Cadena Rey MD*
}

Hace quince años inició actividades la Facultad de Medicina como parte integral de la Fundación Universitaria de Ciencias de la Salud, inspirada en los principios de la Sociedad de Cirugía de Bogotá que fue gestora, no solo de la Fundación, sino también de la naciente facultad.

La vocación docente asistencial ha estado presente en los ideales de la Sociedad desde su creación en 1902. Cuando los diez fundadores se reunieron en el Club Médico que ellos habían constituido dos años antes, plasmaron mediante escritura pública la intención y los aportes en dinero que en forma generosa hicieron, con el fin de construir un hospital moderno al estilo de los mejores del mundo de la época, que ellos conocieron como estudiantes que fueron en algunos centros de Europa y Estados Unidos.

Las primeras reuniones de la Sociedad las dedicaron a la presentación y discusión de casos clínicos y quirúrgicos, que alternaron con la elaboración del magno proyecto de la construcción del nuevo hospital, con el fin de ayudar a resolver la grave crisis de salud causada por la incapacidad del único hospital existente, el de San Juan de Dios, que estaba agobiado por la falta de recursos económicos y científicos, que le impedían atender en forma adecuada a la enorme cantidad de pacientes. Muchos morían en los corredores sin posibilidad de tratamiento.

El 8 de febrero de 1925 abre las puertas el Hospital de San José para hasta hoy nunca cerrarlas. Con ello se brindaría una asistencia de avanzada con personal

* Director-Editor revista Repertorio de Medicina y Cirugia. Miembro de la Sociedad de Cirugía de Bogotá médico actualizado y equipos de apoyo novedosos en aquel entonces. Todo ello se enfocaría a la enseñanza moderna de la medicina y la capacitación quirúrgica de los jóvenes médicos que no disponían de recursos para trasladarse a los países que ostentaban el liderazgo científico.

Diez años después el hospital había cumplido la misión docente encomendada por los fundadores, creando las categorías de Interno Junior e Interno Senior adscritos a las secciones de clínica quirúrgica, clínica médica, ginecología, ortopedia y oftalmología. Era el nacimiento de las especialidades medicoquirúrgicas. Pero no solo era necesario capacitar a los médicos, era fundamental elevar la competencia y conocimientos del personal de enfermería que compartía la asistencia con los Hermanos Hospitalarios de San Juan de Dios y después con las Hermanas de La Presentación de Tours. Para lograrlo, la Sociedad de Cirugía se asoció con la Universidad Nacional de Colombia para crear la Escuela de Enfermeras, que después sería trasladada a la sede de la Nacional en la ciudad blanca y continuaría como facultad hasta nuestros días. Años más tarde la Sociedad erigió su propia facultad de enfermería, que con el prestigio dado por la acreditación y luego reacreditación de alta calidad de su programa académico, hoy se destaca entre las más importantes del país.

Como la razón de ser del Hospital era la actividad quirúrgica, la Sociedad fundó la primera escuela de ayudantes de cirugía, que a través del tiempo se transformaría en la actual Facultad de Instrumentación Quirúrgica. También intentó la creación de una escuela de auxiliares de enfermería, que a la postre se plasmó en un convenio con el SENA y así se convirtió en la sede de la escuela por varios años. Hoy el SENA la continúa en otras instituciones. 
Preocupados por la formación médica de pregrado, los miembros de la Sociedad vieron la necesidad de crear su propia facultad e inclusive se nombró un Decano encargado de organizarla. Por esa época, a mediados del siglo pasado, la Universidad Javeriana fundaba su facultad de medicina, pero ante la carencia de centros de práctica propios, firmaron convenios con La Samaritana y San José, que se convirtieron en hospitales sedes de la naciente facultad y con ello se enterró el proyecto de la facultad propia.

Cuando el Hospital de San Ignacio entró en funcionamiento en la década de los sesenta, la Javeriana trasladó hacia allá los estudiantes que venían a San José y así se creó de nuevo la necesidad de tener pregrado, puesto que los posgrados progresaban y pertenecían a la Sociedad. Fueron varias las universidades interesadas en acudir al hospital y la selección recayó en el Colegio Mayor de Nuestra Señora del Rosario, cuya facultad de medicina fundada a fines del siglo XVIII y después de múltiples viscisitudes en los períodos de la independencia, la reconquista y la república, cerró sus puertas en el siglo XIX.

Con esta alianza entre el Rosario y la Sociedad de Cirugía de Bogotá se reabrió el programa de medicina que fue exitoso desde su iniciación, acudiendo al llamado jóvenes bachilleres de gran desempeño académico que le dieron lustre al proyecto educativo. Pero de nuevo surgieron nuevos vientos que llevaron al Rosario a establecerse en otros centros asistenciales de la ciudad y el Hospital de San José, universitario por vocación, ve de nuevo sus pabellones sin el motor progresista de los estudiantes de pregado. Como siempre, los posgrados continuaron en el hospital, ya que el registro correspondía a la Sociedad.

En 1997 la Asamblea de la Sociedad acogió el proyecto del Presidente Darío Cadena y la Junta Directiva y autorizó la creación de la Facultad de Medicina propia, anhelo que no se había podido cumplir. La asociación con una universidad externa ya había cumplido su ciclo con la Nacional, la Javeriana y el Rosario, terminando siempre con el traslado de los estudiantes a las sedes propias o a otras instituciones mediante contrato, lo cual había obligado al hospital y a los miembros de la Sociedad a adaptarse a nuevos horizontes cada vez que esto sucedía. La infraestructura física y el entorno se venían deteriorando en forma progresiva y se corría el peligro de que se apagara el faro de luz que los fundadores habían entregado a las generaciones siguientes.

La Junta Directiva fortaleció la Fundación Universitaria de Ciencias de la Salud, creada veinte antes por la Sociedad. Contaba con las facultades de enfermería, instrumentación quirúrgica y citohistología. Era el momento justo de crecer y engrandecer la institución docente empleando su radio de acción en el área de la salud. La elaboración del proyecto fue una carrera contra el tiempo y se logró presentar ante el ICFES y el Ministerio de Educación a finales del segundo semestre de 1997. A mediados de diciembre, se recibió la autorización gubernamental para abrir la facultad de medicina. No se ahorraron esfuerzos para terminar la dotación del anfiteatro y los laboratorios de ciencias básicas, las aulas y la contratación del recurso humano en cuanto a docentes y personal administrativo. Así, pudimos abrir las puertas y recibir al primer grupo de estudiantes que iniciaron su carrera profesional el primer semestre de 1998.

En estos quince años la facultad se ha consolidado como una de las mejores del país por su calidad académica, la excelencia en la docencia con profesorado de la más alta calidad y el recurso físico del viejo pero moderno Hospital de San José.

Merece especial mención la autorización que la Asamblea de la Sociedad de Cirugía hiciera al presidente Jorge Gómez, al rector de la Fundación y a los miembros de la Sociedad pertenecientes al Consejo Superior de la universidad, para adquirir a través de la FUCS el antiguo Hospital Infantil Universitario Lorencita Villegas de Santos, cerrado durante una década, saqueado y en el abandono. La reconstrucción y modernización de la vetusta estructura lo convirtieron en uno de los hospitales bogotanos más atractivos y su prestigio ha logrado posicionarlo en sitio de privilegio en esta primera década del siglo XXI. Hoy es sede de nuestra pujante Facultad de Medicina que se vanagloria de la prestante calificación de acreditación de alta calidad 
que confiere el Ministerio de Educación Nacional. De la misma manera nuestros hospitales de San José e Infantil Universitario de San José ostentan igual categoría de acreditación de alta calidad otorgada por el Ministerio de Salud.

El alto nivel que distingue a la facultad se debe a tantas personas que han influido en su desarrollo, que es imposible nombrarlas sin el peligro de caer en omisiones involuntarias. La Sociedad de Cirugía de Bogotá y todo el equipo humano que conforma la Fundación Universitaria de Ciencias de la Salud y los dos hospitales sedes, merecen el reconocimiento general por su dedicación, estudio, sensibilidad social y sobretodo el amor por las instituciones que le dan brillo a la medicina nacional. Recordamos con agradecimiento perenne a quienes me acompañaron en la iniciación de esta exitosa odisea académica: Gladys León incomparable abogada y amiga, Luis Carlos Taborda vicepresidente de la Sociedad, Eduardo Palacios rector, Francisco Barreto vicerrector, Roberto Jaramillo decano, Sergio Parra secretario académico y Raúl Barrios director del Hospital de San José.

Es nuestro deseo e ilusión que este proyecto educativo junto con las instituciones asistenciales perduren en el tiempo y sigan ocupando el lugar de privilegio que les corresponde.

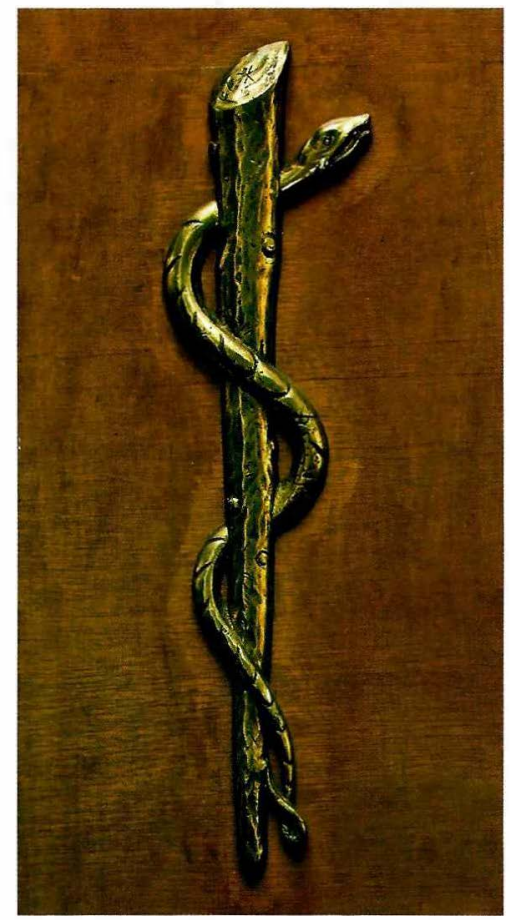

